\title{
Design of Control System of Reconstituted Tobacco Flexible Production Line Based On Industry Control Network
}

\author{
Li Yuliang \\ Engineering and Equipment Division \\ Hangzhou Cigarette Factory, ZJTI \\ Hangzhou, China \\ e-mail: liyuliang@zjtobacco.com \\ Lou Weidong \\ Engineering and Equipment Division \\ Hangzhou Cigarette Factory, ZJTI \\ Hangzhou, China \\ e-mail: louwd@zjtobacco.com
}

\author{
Jin Wenliang \\ Engineering and Equipment Division \\ Hangzhou Cigarette Factory, ZJTI \\ Hangzhou, China \\ e-mail: Jinwl@zjtobacco.com \\ Zhao Yunfeng \\ Engineering and Equipment Division \\ Hangzhou Cigarette Factory, ZJTI \\ Hangzhou, China \\ e-mail: zhaoyf@zjtobacco.com
}

\begin{abstract}
In order to avoid the shortcoming of reconstituted tobacco and the natural tobacco mixed producing method, and adapted to the new cigarette technology, with the designing of the production line's 3 layer network structure and research on realizing method of each layer, the production line based on industry control network was built. The results show that the production line's capacity reaches the level of $2000 \mathrm{~kg} / \mathrm{H}$, and the goal of controlling the percentage of moisture of final product between $12 \%-13 \%$. Through analyzing the experiment results, the industrial control network performance is verified.
\end{abstract}

Keywords-Reconstituted Tobacco; Flexible; Industry Control Network; Programmable Logic Controller(PLC); Communications

\section{INTRODUCTION}

Reconstituted tobacco is one of the main raw material of cigarette, which can effectively improve tobacco raw materials comprehensive utilization, and have rapid response to the personalized, customized and functional requirements for, and also is conducive to reducing tar cigarette products for harm reduction, etc. traditional processing method by mixing Reconstituted tobacco and natural tobacco together has lots of shortcomings [1]. So, in order to avoid the shortcomings and adapted to the new cigarette processing technology, the concept of individual reconstituted tobacco cutting line is created. This kind of production line can overcome the shortcoming of traditional mixed processing method and provide the reference for the future cigarette manufacturing process.

Due to the high stability, large amount of information and the real-time performance of the individual reconstituted tobacco flexible production line, we use IPC as the supervisor and host computer, with the software programmed by Siemens Simatic WinCC 7.0 and Borland Delphi 7.0. For the control of the field device, we use Siemens S7-400 PLC as the main controller, with the software programmed by Simatic Step7 v5.5. Considering the real-time performance, we use Profibus-DP and ethernet protocol as the communication protocol, with the purpose of realizing the function of fieldbus device remote operating, status monitoring, data collecting and system warning.

\section{SYSTEM STRUCTURE}

A typical flexible manufacturing system is generally consists of three subsystems which are processing system, logistics system, control and management system [2]. In order to realize the organic combination between these three subsystems, smooth flow of information supported by the suitable design, build and implementation of industrial control network is the decisive factor. industrial control network described in this paper especially refers to Siemens industrial control network, the whole individual reconstituted tobacco flexible production line is based on Siemens industrial control network.

\section{A. Siemens Industrial Control Network Overview}

As one of the leader in automatic system field, Siemens provides a totally complete industrial communication solution, which obeys 3-layer network structure [3]. It will be described as follows:

Siemens industrial control network is a kind of communication network which covers the whole area of a factory. It is consist of industrial ethernet(IEEE 802.3, $802.3 \mathrm{u}$ ), industrial mobile communication, PROFIBUS, AS-I, EIB and so on. A Typical Siemens industrial control network is described as Fig. 1.

\section{B. Control System Structure of the Reconstituted Tobacco Flexible Production Line}

This line is consist of device-layer, supervisor-layer, management-layer, referring to the Siemens industrial control network 3-layer structure. 


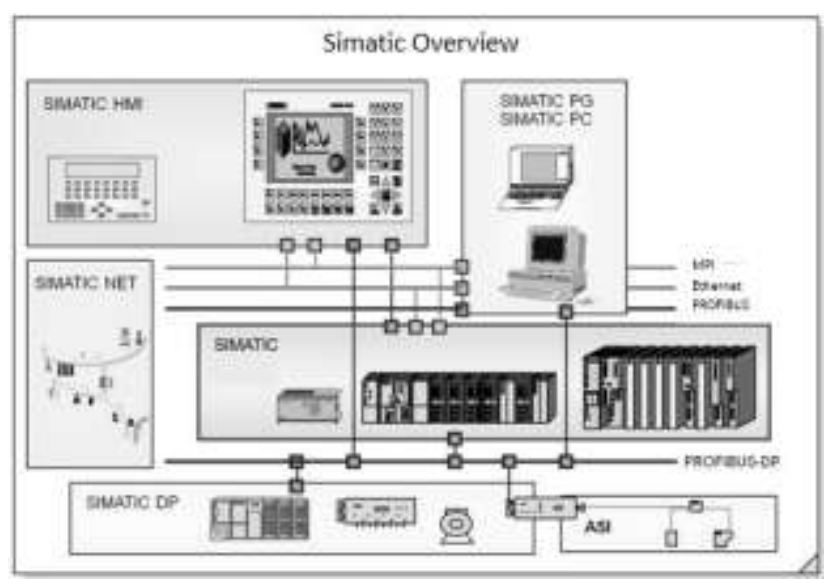

Figure 1. Typical Siemens industrial control network overview.

- Device-layer is consist of several subsystems as follows:

1) Auxiliary device control subsystem

2) Dust-extraction subsystem

3) Tobacco cutting machine subsystem

4) Tobacco dryer control subsystem

5) RFID subsystem

6) Moisture meter system

7) Electrical belt weigher subsystem

8) Tobacco case packing subsystem

- Supervisor-layer contains two IPC which are redundant and in charge of supervisor the subsystems and central operating.

- Management-layer is integrated with the function of web publishing, production data acquisition, production data querying and analyzing.

\section{Control network Structure and communication method}

- Device-layer

In order to realize the real-time synchronized control and high reliability, we use distributed structure in the device-layer based on Profibus-DP protocol which has better real-time performance than traditional Ethernet and is especially suitable for I/O control. The main controller of the devicelayer we selected is Siemens S7-400 PLC which is the master station for the distributed DP slave stations. The distributed DP slave stations contain auxiliary device control subsystem, dust-extraction subsystem, tobacco dryer control subsystem, RFID subsystem and moisture meter system which are connected with each other by the Profibus-DP network.

For the subsystems integrated with a small individual controller, like electrical belt weigher subsystem and tobacco case packing subsystem which are called intelligent DP slave station, master station directly communicates with them by the method of "master-slave" communication based on Profibus-DP

- Supervisor-layer

The main function of this layer is data supervisor, also it directly collects data from master PLC based on industrial ethernet. The software is developed by Simatic WinCC 7.0.

- Management-layer

The central control IPC acquires all kinds of data from supervisor-layer by the way of OPC. This OPC server is supplied by the supervisor-layer, while the client is management-layer. The background database is MS-SQL SERVER, the software is developed by Borland Delphi 7.0.

The network topology is described as Fig. 2.

\section{REALIZATION OF NETWORKS'COMMUNICATION}

Fig. 2 shows the structure of the flexible cutting line networks and the networks at different layers. It also shows that the configuration and programming for such networks are realized by using different means of communication. By this way, the communication among all subnet system and networks at all layers are realized. Siemens STEP7 is adopted in device-layer as the configuration and programming software; WinCC is adopted in supervisor-layer; Managing data collection is reached by industrial data bridge and management software is developed by Borland Delphi [5].

\section{A. Realization of Device-layer networks' communication}

The structure of controlling networks for device-layer controlling system adopts main PLC as master station and distribution I/O station as salve stations. The hardware relationship among them is configured by Siemens STEP7. The system's hardware configuration is shown in Fig. 3.

The Fig. 3 shows that the master station has three kind of slave station: one is ET200S slave station, one is Danfoss frequency converter and moisture meter slave station; the other slave station is the PLC controller like Siemens S7-300 of other system which are called intelligent salve station.

- The communication between master station and distributed I/O salve station

Normally the communication between them we can directly call Siemens STEP7 system function SFC14 and SFC15 in the programming procedure.

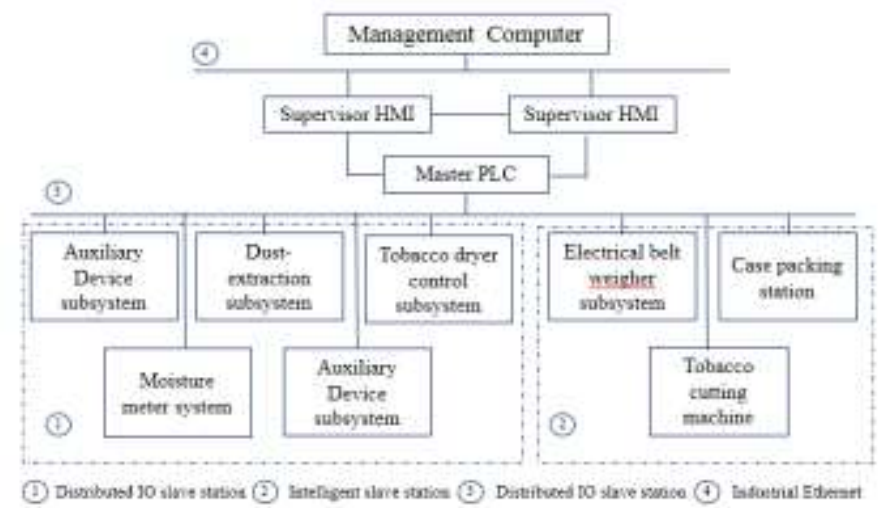

Figure 2. System Structure. 
- The communication between master station and intelligent salve station

The communication between master station and intelligent salve normally is based on Profibus-DP, and its realization is totally different from the communication between master station and distributed I/O salve station. The procedures are described as follows [5]:

1) In the hardware configuration of the master station's project file, an intelligent salve station must be configured.

2) The operation mode of the DP port for the intelligent salve station must be configured as slave mode

3) Configure data communication memory area allowed by intelligent salve station.

4) In network configuration, drag intelligent salve station onto master station's Profibus-DP network for main station. This is shown in Fig. 3.

5) Couple the master station's PLC and intelligent slave station's PLC;

6) Mapping the communication data memory area of the configured intelligent slave station.

With the steps described above, the communication between the master station and intelligent Slave station is established.

\section{B. Realization of the Supervisor-layer's Network Communication}

The supervisor-layer uses Siemens Hmi configuration software WinCC, which is well-developed and can provide totally perfect communication support for Siemens series PLC. WinCC can build connections between the communication of various kinds of Siemens PLC. Very conveniently through a built-in SIMATIC S7 protocol suite drive system via all kinds of network access protocols, such as industry Ethernet, Profibus-DP, MPI and TCP/IP, etc.

\section{Realization of the the Management-layer's Network Communication}

According to the general function design of modern automation system's management layer's management PC ,it should has the following two basic functions: one is storing production process data as well as product information and the other is analyzing data.

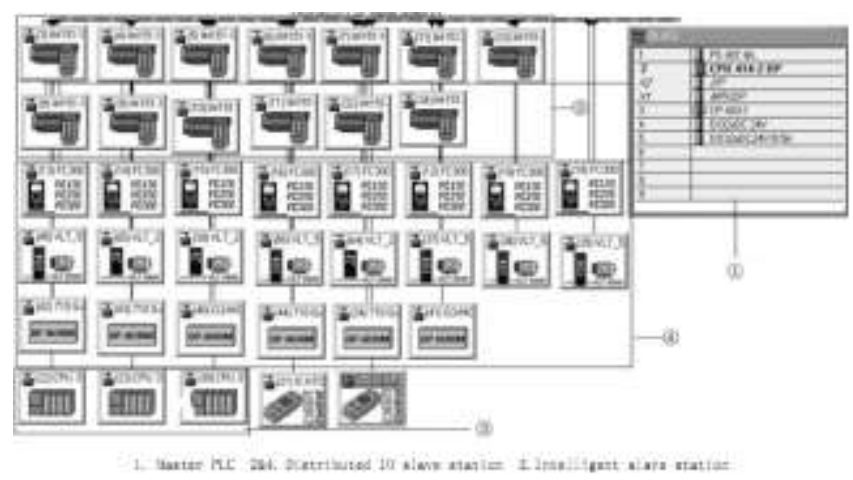

Figure 3. System Hardware Configuration.
- The realization of productive process data and product information storage function based on industrial Ethernet.

In this project, our management IPC in the management-layer uses OPC client through industrial Ethernet to acquire default data which is provided by the supervisor-layer's software WinCC as OPC server[8], after which it will record the data required by WinCC from the master PLC into database by using WinCC IDB(Industrial Data Bridge). IDB has the following characteristics [6]:

1) It can be used as data source as well as data destination.

2) It can be connected to many SCADA systems produced by different manifactures with different kinds of OPC interfaces.and it makes data archiving more convenient by putting WinCC Duntime's realtime data into the history database.

3) It can connect the automation layer's data to the IT layer.

The management PC acquires and stores WinCC OPC server's data into background database on industrial Ethernet through the following procedures: after running WinCC Runtine, the OPC server will start. Then, IDB will take this OPC server's data as source data and unload them to the data destination such as ....after corresponding programming and setting.

- The realization of management-layer client We develop a data querying software based on history database by Delphi 6.0 and realize the functions such as querying by product information keywords, analyze and making statistics exporting during different time periods to excel forms and printing out report forms, etc.

\section{CONCLUSION}

The primary line reconstituted tobacco flexibility control system described in this article uses Siemens industrial controlling network architecture as its communication infrastructure and Siemens PLC as its central controller. It realizes network communication between different layer's through Profibus-DP network, adopts Siemens configuration software WinCC as its supervisor layer software to realize network communication between the supervisor-layer and devicelayer with TCP/IP protocol and realizes the production data storage in the management-layer through industrial Ethernet in the form of OPC. This kind of design, which is in correspondence with Siemens Total Integrated Automation standard is a relatively advanced controlling strategy in industrial control and is suitable to the integration of various industrial control network-based controlling system. Thus, it has a promising supplication prospect. This industrial control network based reconstituted tobacco flexible production line can reach the production capacity of $2000 \mathrm{~kg} / \mathrm{h}$ and realize the output moisture target to be $12-13 \%$.

This line can also improve the quality and effective availability of paper-making reconstituted tobacco thread 
and provide a strong guarantee of primary line group processing, new technique pattern of flexible production as well as the reduction of harmful components in the smoke.

\section{ACKNOWLEDGMENT}

The authors would like to acknowledge the support of funding (Grant No. ZJZY2013E016) from Hangzhou Cigarette Factory, Zhejiang Tobacco of Industry, China.

\section{REFERENCES}

[1] Wang Na and Wang Dingwei, "Feasibility of Industry Application of Paper-making Tobacco," Journal of Anhui Agricultural Sciences China, vol. 40, pp. 15900-15902, August 2012.

[2] Li Tao, Guan Zhong, and Ma Lu, "Preliminary Discussion on Flexible Manufacturing in Tobacco Primary Processing," Tobacco Science and Technology China, vol. 233, pp. 20-25, December 2006.

[3] Cui Jian, Li Jia, and Yang Guang, "Guide To Siemens Industry Communication," China Machine Press.

[4] Huang Zhen, Lv Jinsong, and Zeng Eryang, "Configuration of OPC-based Distributed Control System," Tobacco Science and Technology China, vol. 300, pp. 32-35, July 2012.

[5] Siemens Automation and Drives, "Helping Document with STEP 7," 2011.

[6] Siemens Automation and Drives, "Helping Document with Industrial Data Bridge,” 2012.

[7] Lin Yingyun, Yang Yupu, "Two Methods to Realize Serial Communication based on WinCC," Electronic Measurement Technology, vol. 29, pp. 115-116, February, 2006.

[8] Rao Keke and Li hua, "The communication between WinCC and Multi-Client System," "Chemical Engineering Automation and Instrument", vol.39, pp. 133-135, January, 2012.

[9] Li Yuliang, Fang Boyang, Qian Yongan, Yuan Genhong, "Profibus-based RFID Applying Control System for Cut Tobacco Case Filling," Tobacco Science and Technology China, vol. 293, pp. 14-16, November 2011

[10] Su Mei, Wang Zhongmin, "Research And Application On OPC Data Access Server," Micro Computer Information, vol. 22, pp. 11-13, July, 2006. 